\title{
CAPÍTULO VII \\ DIAGNÓSTICO DE LAS HABILIDADES INVESTIGATIVAS DE LOS ALUMNOS DE LICENCIATURA EN CONTADURÍA PÚBLICA DEL CUCSUR - UDG
}

\section{María Luz Ortiz Paniagua}

Doctora en Contabilidad y Finanzas, Profesora Titular A del Centro Universitario de la Costa Sur de la Universidad de Guadalajara, Jalisco México. Investigadora SNI Nivel I. ortizpan@yahoo.com.mx.

\section{Luís Carlos Gámez Adame}

Doctor en Contabilidad y Finanzas, Profesor Titular C del Centro Universitario de la Costa Sur de la Universidad de Guadalajara, Jalisco México. Responsable Cuerpo Académico UDG - 733. cgamez64@yahoo.com.mx.

\section{Roberto Joya Arreola}

Doctor en Contabilidad y Finanzas, Profesor Titular A del Centro Universitario de la Costa Sur de la Universidad de Guadalajara, Jalisco México. Investigador SNI Nivel I. robertojoyaarreola@yahoo.com.mx.

\section{Resumen}

La valoración del papel del contador público en la actualidad resulta una tarea compleja, donde es necesario tomar en consideración su aporte al desarrollo de las organizaciones y su función orientada al cuidado de los intereses de la sociedad. En este contexto, constituye un imperativo para este profesional en el ejercicio de sus funciones dominar la metodología de la investigación científica. Pero, ¿se encuentran suficientemente preparados en este aspecto los alumnos de Licenciatura en Contaduría Pública del Centro Universitario de la Costa Sur de la Universidad de Guadalajara?; en este sentido fue el estudio realizado, cuyo propósito fundamental consistió en diagnosticar las causas que provocan que los alumnos de la carrera de Licenciatura en Contaduría Pública de este centro universitario posean pocas habilidades investigativas. La metodología aplicada corresponde a una investigación causal que busca entender cuáles variables son las causantes del efecto estudiado, identificando la relación funcional entre causa y efecto. Se trata de un tipo de investigación experimental y estadística, donde se utiliza la técnica de triangulación ampliamente utilizada en la investigación científica, aplicándose encuestas a docentes y alumnos de los semestres terminales, para posteriormente triangular los resultados obtenidos. Las encuestas se procesaron por estadísticos descriptivos y pruebas de fiabilidad. Los principales resultados obtenidos muestran limitaciones en el diseño curricular, la forma de enseñanza, la realización de investigaciones en materias del ejercicio profesional de la carrera y la actitud de los alumnos como causas de que los alumnos de esta carrera posean pocas habilidades investigativas, planteándose como conclusión fundamental la imperativa necesidad de que los maestros desde la didáctica de sus materias motiven a sus alumnos a la realización de investigaciones.

Palabras clave: Habilidades investigativas, perspectiva alumnos, perspectiva docente. 


\title{
DIAGNOSIS OF THE INVESTIGATIVE SKILLS OF THE DEGREE OF DEGREE IN PUBLIC DEBT OF THE CUCSUR - UDG
}

\begin{abstract}
The assessment of the role of the public accountant at present is a complex task, where it is necessary to take into account their contribution to the development of organizations and their role oriented to the care of the interests of society. In this context, it is an imperative for this professional in the exercise of his functions to master the methodology of scientific research. But are students in the Public Accounting Department of the University Center of the South Coast of the University of Guadalajara sufficiently prepared in this regard? in this sense, it was the study carried out, whose main purpose was to diagnose the causes that cause the students of the Degree in Public Accounting of this university center to have few investigative skills. The applied methodology corresponds to a causal investigation that seeks to understand which variables are the cause of the studied effect, identifying the functional relationship between cause and effect. It is a type of experimental and statistical research, where the triangulation technique widely used in scientific research is used, applying surveys to teachers and students of the terminal semesters, to later triangulate the results obtained. The surveys were processed by descriptive statistics and reliability tests. The main results obtained show limitations in the curricular design, the form of delivery, the realization of research in subjects of the professional exercise of the career and the attitude of the students as causes of which the students of this race possess few investigative abilities, posing as fundamental conclusion the imperative need that teachers from the didactics of their subjects motivate their students to conduct research.
\end{abstract}

Keywords: Research skills, student's perspective, teaching perspective.

\section{Capitulo resultado de proyecto de investigación culminado}

Las Habilidades investigativas de los alumnos de Licenciatura en Contaduría Pública del CUCSUR - UDG. Proyecto concluido.

\section{Introducción}

Las universidades deben desarrollar capacidades para la investigación en los estudiantes e incorporar la investigación como estrategia de enseñanza aprendizaje en el currículo. Un estudiante con las competencias básicas en investigación es capaz de plasmar de manera clara y precisa los problemas de su entorno, delimitarlos, analizarlos y desde su disciplina proponer estrategias de mitigación a esa situación generando un aporte a la sociedad. (Hernández, Barbera, Vega \& Bru, 2018, p. 149)

Desarrollar habilidades investigativas en los alumnos de Licenciatura en Contaduría Pública (LCP) ayuda a describir las causas de algún acontecimiento en particular, es un 
proceso que permite obtener nuevos conocimientos y aplicarlo creativamente a la realidad donde se ejercerá la profesión, posibilitando la realización de diagnósticos profundos y argumentados de la situación de una organización, ya sea para solucionar problemas o realizar algunas modificaciones, proponer soluciones y fundamentar las propuestas, entre otros.

Sin embargo, se reconoce por observación realizadas que los alumnos de LCP del Centro Universitario de la Costa Sur de la Universidad de Guadalajara (CUCSUR - UDG) arriban a la materia de Seminario de Titulación y no saben elaborar de forma independiente un proyecto de investigación, realizar solos una investigación de campo de baja complejidad, requiriendo asesoría constante de los maestros que imparten esta materia; por lo cual es necesario buscar las causas que provocan que durante la carrera no se desarrollen plenamente las habilidades investigativas y por ende la pobre motivación que existe a titularse por tesis. Lo expresado, muestra una contradicción entre la necesidad que el contexto actual impone a los contadores, de no ser sólo suministradores de información, sino de ser entes activos de la gestión organizacional y la poca habilidad de los alumnos de LCP del CUCSUR - UDG en metodología de la investigación, lo que constituye el problema de investigación.

A partir de los elementos planteados, la pregunta problema fue ¿Cuáles son las causas que provocan que los alumnos de 6to, 7 mo y 8 vo semestre de la carrera de Licenciatura en Contaduría Pública (LCP) del CUCSUR - UDG posean pocas habilidades investigativas?

Planteándose como objetivo general diagnosticar las causas que provocan que los alumnos de 6to, 7mo y 8vo semestre de la carrera de LCP del CUCSUR - UDG posean pocas habilidades investigativas.

Para la realización de la investigación y a partir de la aplicación de una técnica grupal se determinaron las siguientes cuatro variables fundamentales a valorar desde la perspectiva de docentes y alumnos: El diseño curricular, la forma de enseñanza, la realización de investigaciones en materias del ejercicio profesional de la carrera y la actitud de los alumnos.

El alcance espacial está delimitado a los alumnos de 6to, 7mo y 8vo semestre de la carrera de Licenciatura en Contaduría Pública del CUCSUR - UDG y los docentes de esta carrera, mientras que el alcance temporal fue el ciclo $2018 \mathrm{~B}$.

Las principales limitaciones del estudio se relacionan con la posibilidad de que los sujetos participantes en el estudio no respondan con total sinceridad las preguntas de los instrumentos utilizados, por lo cual se realizó una prueba piloto donde se determinó la prueba de fiabilidad del Alpha de Cronbach.

En el ámbito epistemológico se asume como un antecedente consultado la investigación desarrollada por Hernández et al. (2018) relacionada con "El aprendizaje basado en el problema: una estrategia pedagógica para la formulación de proyectos de investigación en el aula", mientras que en el ámbito local le antecede el estudio realizado en el Departamento de Contaduría del Centro Universitario de la Costa Sur de la Universidad de Guadalajara por su Colegio Departamental para explicar la baja eficiencia de los Exámenes Generales para el Egreso de Licenciatura (EGEL) del Centro Nacional de Evaluación para la Educación Superior (CENEVAL) que presentan los alumnos de la LCP, que posibilitó el establecimiento de algunas estrategias a nivel departamental y por academia. Estos antecedentes, posibilitó al Cuerpo Académico UDG - 733 continuar con el estudio que hoy se presenta. 


\section{Fundamentación teórica}

La investigación es una función fundamental de la universidad; constituye un elemento importante en el proceso educativo porque a través de ella se genera conocimiento y se propicia el aprendizaje para la generación de nuevo conocimiento; además, la investigación vincula la universidad con la sociedad. Al ser una función fundamental, sustantiva, la investigación es un deber ser (Areválo, Miranda, Galindo \& Morgan, 2004; p.119). Por tal razón, la investigación científica es muy importante en la formación del contador, ya que a través de ella se obtiene información de manera clara, precisa y fiable, sobre cuya base se toman decisiones.

Porlan (1992) agrupa en cuatro los modelos pedagógicos: Tradicional, tecnológico, espontaneísta o activo y constructivista o alternativo-investigativo (como se citó en Orozco, Sosa y Martinez, 2018, p.450)

El modelo tradicional está centrado en el docente y los contenidos, donde el contexto y el alumnado queda en un segundo plano; siendo el conocimiento una selección de lo producido por la investigación científica a criterio del docente.

El modelo tecnológico armoniza en cierta medida una preocupación de transmitir el conocimiento aplicando métodos activos, donde se involucra la teoría y la práctica.

El modelo espontaneísta potencia la inserción del alumno en la realidad que le rodea, donde se considera más importante desarrollar en el alumno habilidades de observación y búsqueda de información que el propio aprendizaje de los contenidos.

Se coincide con López \& Hernández (2016, p.575) conque la educación está en un proceso de renovación radical por influencia de la Tecnología de la Información y la Comunicación (TIC), particularmente por exigencia de los nativos digitales y de las nuevas circunstancias sociales y culturales. Pese a ello, en la Pedagogía todavía no se ha creado un modelo que asuma a estas herramientas en la formación humana de manera satisfactoria y consensuada. Básicamente se utilizan como medios para ayudarse en el proceso instruccional.

El entorno actual caracterizado por el desarrollo de las redes sociales, la internet y las tecnologías de la información han provocado que los docentes no sean los únicos con acceso a nuevos conocimientos, lo que provoca un cambio en la concepción de los modelos educativos, dando paso a los modelos constructivistas o alternativo investigativo, donde bajo la orientación del maestro el alumno construye su propio conocimiento a partir de la solución de problemas. En este contexto, es indispensable que los alumnos tengan la capacidad de utilizar herramientas y técnicas de investigación; y esta tarea le corresponde no sólo a la materia de metodología de la investigación, sino que debe ser el trabajo coordinado de los docentes que imparten las materias del ejercicio profesional.

A lo anterior se añade que el Licenciado en Contaduría Pública debe enfrentarse a un contexto empresarial donde "el conocimiento es primordial para el desarrollo económico, por lo que las empresas desarrollan cada vez más su habilidad para explotar los recursos y capacidades valiosas, esto a su vez lo traducen en la concentración de sus esfuerzos en formalizar el conocimiento tácito en un conocimiento explícito a través de la aplicación de actividades desarrolladas en manuales o procedimientos que le permiten enfrentar la 
velocidad con la que se presentan los cambios tecnológicos. La empresa en cuestión se caracteriza por poseer el conocimiento enfocado a la innovación, en nichos de mercado muy especializados y con alta aplicación de investigación y desarrollo tecnológico". (Tiburcio, Godínez \& Aguilar, 2018, p.3)

Por lo que se coincide con Infante (2014, p.918) conque el panorama actual del proceso enseñanza-aprendizaje plantea nuevos retos académicos, especialmente en lo que se refiere a metodologías capaces de construir competencias orientadas al logro de una mayor autonomía del estudiante, puesto que el aprendizaje será más efectivo si en alguna etapa de la experiencia el alumno puede participar activamente mediante la experimentación, el análisis y la toma de decisiones y con Caro (2014) cuando explica que en la actualidad la educación superior se enfoca en el desarrollo de competencias y el desarrollo tecnológico que conllevan a cambios significativos en las estrategias didácticas, las cuales deben adaptarse a las nuevas necesidades de manera coherente y pertinente.

Lo cual es ratificado por Hernández el al. (2018, p.148) cuando afirman que la tarea consiste en formar estudiantes capaces de analizar y hacer frente a los problemas cotidianos, valorando e integrando el saber que los conducirá a la adquisición de competencias profesionales.

Por su parte López (2001, p.30) aporta un concepto de habilidad de investigación definiéndola como una manifestación del contenido de la enseñanza, que implica el dominio por el sujeto de las acciones práctica y valorativa que permiten una regulación racional de la actividad con ayuda de los conocimientos que el sujeto posee, para ir a la búsqueda del problema y a su solución por la vía de la investigación científica. Lograr estas habilidades es un reto de la educación superior en este siglo.

Martínez \& Márquez (2014, p.350) al analizar diferentes definiciones de habilidad investigativa la sistematizan en cuatro grupos genéricos:

- Las habilidades investigativas representan un dominio de acciones para la regulación de la actividad investigativa.

- Las habilidades investigativas representan un conjunto de habilidades que pudieran considerarse como invariantes de la actividad investigativa.

- Las habilidades investigativas representan un dominio del contenido de la enseñanza investigativa o lo que sería igual, de su sistema de conocimientos, hábitos, valores y actitudes.

- Las habilidades investigativas representan una generalización del método de la ciencia.

Hoy se enfatiza que no hay verdadera educación superior sin actividad de investigación explícita e implícita, ella forma parte del proceso enseñanza-aprendizaje y tiene un gran valor en la formación profesional. La investigación constituye un proceso contextualizado, por lo que no la podemos ver aislada, sino inserta en problemáticas globales, laborales; se debe concebir en una relación directa con los problemas que vive la sociedad. Se investiga para transformar la realidad y con ello contribuir al desarrollo humano y por lo tanto mejorar la calidad de vida, por lo que ella se constituye en un medio muy valioso para lograr cualquier transformación en el ámbito profesional. (Montes de Oca \& Machado, 2009, p.3) 


\section{Metodología}

El estudio que se presenta, a partir de Chawla \& Sodhi (2011). es una investigación causal que busca entender cuáles variables son las causantes del efecto estudiado, identificando la relación funcional entre causa y efecto. Se trata de un tipo de investigación experimental y estadística, donde se utilizan procedimientos para la reducción de los datos como la determinación de parámetros de posición (moda y media), conjuntamente con la técnica de triangulación. El procedimiento diseñado está compuesto por los siguientes pasos:

1. Determinación de las causas del poco desarrollo de las habilidades investigativas desde la perspectiva de los docentes.

2. Determinación de las causas del poco desarrollo de las habilidades investigativas desde la perspectiva de los alumnos.

3. Triangulación de los resultados

Para el tratamiento de las muestras se aplicó muestreo aleatorio simple (docentes) y estratificado (alumnos). Los resultados de las encuestas aplicadas se procesan por SPSS 23.0

Antes de aplicar las encuestas se realizó una prueba piloto a los alumnos y docentes para valorar la fiabilidad de los instrumentos diseñados, donde se crearon dos grupos pilotos de 22 y 10 individuos respectivamente, donde al procesar por el Alpha de Cronbach se obtuvo 0.823 y 0.857 respectivamente. Estos resultados a partir de Huh, Delorme \& Reid (2006) se consideran satisfactorios, por lo que los instrumentos diseñados son fiables.

Para el desarrollo del diagnóstico se aplicó muestreo aleatorio simple para los maestros, donde de una población de 37 docentes se encuestó 34 con un nivel de confianza del 95 por ciento y un margen de error del 5 por ciento.

La población de alumnos participantes en el estudio estuvo integrada por una población de 88 alumnos de los semestres sexto, séptimo y octavo, a partir de considerar a los alumnos de estos semestres como población objetivo sobre la base de las siguientes consideraciones:

- Ya han recibido la materia de metodología de la investigación.

- Han cursado materias de todas las disciplinas del ejercicio de la profesión.

- Han recibido las materias de matemáticas, estadísticas e informática que deben capacitarlo en el uso de este instrumental para solucionar problemas profesionales.

Obteniéndose una muestra de 72 alumnos con un nivel de confianza del 95 por ciento y un margen de error del 5 por ciento, configurada en los siguientes estratos: 
Tabla 1. Estratos de la muestra

\begin{tabular}{cc}
\hline Estrato & Cantidad muestra \\
\hline Sexto semestre & 18 \\
\hline Séptimo semestre & 26 \\
\hline Octavo Semestre & 28 \\
\hline Total & 72 \\
\hline
\end{tabular}

Fuente: Elaboración propia (2019).

\section{Procedimiento}

Paso 1. Determinación de las causas del poco desarrollo de las habilidades investigativas desde la perspectiva de los docentes.

a) Identificación de las variables:

Mediante la técnica de trabajo en grupo con los miembros del colegio departamental y el Cuerpo Académico CA UDG - 733 se determinaron las siguientes variables a evaluar:

- Diseño curricular

- Forma de impartición

- Realización de investigaciones en materias del ejercicio profesional de la carrera

- Actitud de los alumnos

A partir de las variables identificadas se elaboró una encuesta para aplicar a los docentes, compuesta por 15 preguntas, con una escala múltiple de tipo razón con los siguientes valores:

6 - siempre, totalmente, si

4 a 5 - valores intermedios que valoran casi siempre, generalmente

1 a 3 - valores intermedios que valoran a veces, pocas veces, casi nunca

0- nunca, no, no sé

Se considera como satisfactorios los resultados entre 4 al 6 .

Las respuestas a las preguntas del cuestionario se procesan por la moda y posteriormente se determina la media aritmética por variable, donde se asume la siguiente escala valorativa:

6 - Excelente

Igual o mayor de 4 y menor de 5 - Bien, satisfactorio

Igual o mayor de 3 y menor de 4 - regular 
Igual o mayor de 1 y menor de 3 - deficiente

0 - mal

Paso 2. Determinación de las causas del poco desarrollo de las habilidades investigativas desde la perspectiva de los alumnos.

De las variables ya identificadas, las factibles de aplicar a los alumnos para su valoración fueron:

- Forma de impartición

- Realización de investigaciones en materias del ejercicio profesional de la carrera

- Actitud de los alumnos

En el caso de la variable diseño curricular, se consideró que los alumnos no poseían los elementos necesarios y los conocimientos requeridos para realizar una adecuada valoración de esta variable.

Posteriormente, se elaboró la encuesta para aplicar a los alumnos de 6to, $7 \mathrm{mo}$ y $8 \mathrm{vo}$ semestre, compuesta por 15 preguntas con una escala múltiple de tipo razón para las preguntas de respuesta cerradas, con los siguientes valores:

6 - siempre, totalmente, si

4 a 5 - valores intermedios que valoran casi siempre, generalmente

1 a 3 - valores intermedios que valoran a veces, pocas veces, casi nunca

0 - nunca, no

Se considera como satisfactorios los resultados entre 4 al 6 .

Las respuestas a las preguntas del cuestionario se procesan por la moda y posteriormente se determina la media aritmética por variable, donde se asume la siguiente escala valorativa:

6 - Excelente

Igual o mayor de 4 y menor de 5 - Bien, satisfactorio

Igual o mayor de 3 y menor de 4 - regular

Igual o mayor de 1 y menor de 3 - deficiente

0 - mal

En el caso de las preguntas abiertas se listan, determinándose la frecuencia; donde se consideran representativas las respuestas con una frecuencia $\geq 60.0$ porciento, asumiendo a Campistrous \& Rizo (1998).

Paso 3. Triangulación de los resultados

Se realiza la comparación de los resultados obtenidos de la aplicación de las encuestas de los maestros y de los alumnos buscando las coincidencias, se asumen aquellos resultados que coincidan en un 100 porciento. 


\section{Resultados}

Al procesarse los resultados de la variable de diseño curricular, a partir de la perspectiva de los maestros, se obtiene una media de 3.33 que evalúa como regular a la variable, dado que los docentes consideran que, aunque el diseño de sus materias en cuanto a contenido le permite el desarrollo de habilidades investigativas, la composición en horas y la interrelación de horas dedicadas a la teoría y la práctica les limita desarrollar habilidades investigativas en los alumnos. Esta variable no fue valorada por los alumnos.

En la variable forma de impartición con una moda de 4 en las tres preguntas realizadas y una media de 4, expresando un resultado satisfactorio, los maestros consideran que explican con claridad y de forma motivante para que los alumnos investiguen, así como consideran que su preparación en metodología de investigación les pone en una situación ventajosa para utilizar estrategias didácticas en sus materias para que los alumnos se incorporen a la investigación.

Mientras los alumnos encuestados evalúan a esta variable con una media de 4 que es satisfactorio con una pregunta evaluada de 5 , otra de 4 y otra de 3 , donde consideran que el mayor problema del proceso de enseñanza está vinculado a que no se les promueve a que desarrollen habilidades para investigar o realizar de forma independiente un proyecto.

La variable realización de investigaciones a criterio de los docentes obtuvo una media de 3 que la ubica como regular, dado por cuatro preguntas con una moda de 3 , una pregunta con la moda de 4 y otra con 2 . En esta variable se muestra un reconocimiento de la planta profesoral de las siguientes limitaciones.

- No orientan investigaciones a sus alumnos.

- Vinculan poco sus materias a proyectos de investigación personal, de cuerpos académicos o departamental que se relacionen con los contenidos de estas.

- No es común que orienten y analicen con sus alumnos artículos científicos.

- No les orientan la presentación de trabajos, artículos, reseñas, entre otros a sus alumnos; exigiéndole la aplicación de la redacción científica y las referencias bibliográficas de acuerdo a una norma específica (APA 6, ISO, Chicago, GOST, etc).

- No es común que asuman como criterio de evaluación en los trabajos orientados a entregar por los alumnos la redacción científica y la forma de referenciar.

Lo que es contradictorio, ya que se consideran adecuadamente preparados en metodología de investigación y por lo tanto, con capacidad para aplicarla en la impartición de sus materias.

Al igual que la variable anterior, esta variable es valorada de satisfactorio (media de 4.33) por los alumnos, destacándose que casi nunca se les orienta la realización de investigaciones de campo, no se les vincula a proyectos de investigación que se desarrollan por la propia universidad $\mathrm{u}$ otras instituciones y tampoco se les exige la redacción científica y referenciar por una norma en la presentación de tareas o trabajos orientados.

En la variable actitud de los alumnos, la media de la variable fue de 2.33. Siendo deficiente y la peor evaluada por los docentes, donde estos consideran que los alumnos: 
- No poseen las habilidades que les permita realizar una investigación de campo.

- Muestran poca motivación con realizar investigaciones de campo, análisis científicos, discusiones grupales, trabajos de indagación teórica, entre otros

- Manifiestan una actitud negativa en aplicar el método científico a su profesión.

Los alumnos evalúan de forma regular esta variable al obtenerse una media de 3.66, donde están de acuerdo que no se sienten motivados con la realización de investigaciones de campo, análisis científicos, discusiones grupales, entre otros, pero reconocen su utilidad e importancia en su formación profesional, lo que consideran puede estar dado porque poseen pocas habilidades de investigación y están totalmente de acuerdo que esta carencia es una de las causas para que decidan no titularte por tesis.

Al triangular los resultados, se considera que las principales causas de que los alumnos no desarrollen sus habilidades investigativas están relacionadas con:

- La cantidad en horas y la composición de horas dedicadas a la teoría y la práctica en los programas de las materias del ejercicio de la profesión que no contribuye al desarrollo de las habilidades investigativas de los alumnos.

- Se orientan pocas investigaciones a los alumnos durante la carrera.

- Poca vinculación de las materias a proyectos de investigación institucionales o de otras instituciones.

- No se exige la aplicación de la redacción científica y las referencias bibliográficas de acuerdo a una norma específica en las tareas orientadas a los alumnos, no siendo parte de los criterios evaluativos.

- Poca motivación del alumnado para realizar investigaciones de campo, análisis científicos, discusiones grupales, trabajos de indagación teórica, entre otros

Al procesarse la pregunta abierta se destacan las siguientes consideraciones de los alumnos por tener una frecuencia $\geq 60$ porciento:

- Los maestros no motivan a hacer una investigación científica.

- Falta de orientación sobre cómo investigar.

- Poca orientación de temas interesantes o que motiven a investigar.

- No saber redactar de forma científica y planear una investigación.

\section{Discusión}

Los resultados obtenidos ponen en la mesa de discusión un grupo de causales que conllevan a reflexiones importantes; indudablemente se evidencia un pobre reconocimiento de los docentes de sus limitaciones, considerando a los alumnos como máximos responsables del pobre desarrollo de sus habilidades investigativas, donde indudablemente no reconocen que desde las materias que imparten pueden diseñarse e implementarse estrategias didácticas para motivar a sus educandos a la investigación científica, a través de la solución de problemas profesionales reales donde deban aplicar el método científico para solucionarlo. 
Es necesario que la planta profesional reconozca que la docencia en la actualidad no puede realizarse con los métodos tradicionales, hoy en las universidades se encuentran transitando las llamadas generaciones milennials y centennials, las cuales se han desarrollados en entornos digitales, provocando que los modelos semipresenciales y a distancia ganen cada vez más espacio, cuya motivación pasa indudablemente por el uso de la tecnología y la creatividad como base del proceso de enseñanza aprendizaje. Ante este contexto, la tendencia del profesorado de culpar a la cantidad de horas como una causal de no vinculación de su materia a la investigación científica, sin asumir que en las nuevas condiciones es necesario pensar como trabajar con los alumnos para motivarlos en habilidades de búsqueda, análisis de información y aplicación creadora del conocimiento, muestra un no reconocimiento de la necesaria transformación educativa.

Lo expresado provoca una llamada a la reflexión interna, a partir de las ideas expresadas por Gutiérrez (2018, p.50), Presidente de la empresa GADEA, premio en gestión de Recursos Humanos del Foro Burgos/Castilla y León 2009 y Presidente del Consejo Social de la Universidad de Valladolid, cuando afirma que la universidad es un muerto viviente porque no hay calidad en el nivel de la enseñanza, la investigación y la transferencia de conocimiento en correspondencia a las necesidades del entorno.

\section{Conclusiones}

La utilización de la investigación científica como parte de las estrategias didácticas de los docentes en la carrera de Licenciatura en Contaduría Pública debe motivar a un aprendizaje consciente y al desarrollo de habilidades investigativas que constituyen una necesidad de este profesionista ante el entorno digital y las exigencias del mercado laboral donde desarrolla su actividad.

En este sentido, el desarrollo de proyectos de investigación desde las diferentes materias o en proyectos integradores de años donde el alumno aprenda haciendo, al mismo tiempo que se desarrolla su capacidad de proponer soluciones desde los postulados teóricos a los problemas actuales de la práctica profesional es un imperativo que no puede seguir esperando. Por consiguiente, este camino debe emprenderse desde los procesos de enseñanza aprendizaje en el aula, donde el docente debe estar consciente de su papel.

Sin embargo, el diagnóstico realizado en la carrera de LCP del CUCSUR - UDG ha identificado insuficiencias tanto en el diseño curricular, la forma de impartición, la realización de investigaciones en materias del ejercicio profesional de la carrera y la actitud de los alumnos, planteándose como un imperativo la necesidad de que los maestros desde la didáctica de sus materias motiven a sus alumnos a la realización de investigaciones.

Esta investigación es un primer acercamiento a esta problemática en el CUCSUR UDG por lo cual se considera pertinente continuar en estudios posteriores profundizando en el tema.

Finalmente, conviene destacar la necesidad de aplicar estrategias didácticas centradas en el estudiante, a partir de la cual el alumno busque su propia información y la contextualice a la realidad de su entorno, desarrollando su capacidad de formular propuestas de soluciones por medio de un proyecto. 


\section{Referencias bibliográficas}

Areválo, J., Miranda, N., Galindo, J. \& Morgan, M. (2004). Los paradigmas de la calidad educativa, de la autoevaluación a la acreditación. Recuperado de http://riev. org/media/Libro Modelo V.pdf

Campistrous, L. \& Rizo, C. (1998). Indicadores e Investigación Educativa. La Habana, Cuba: Instituto Cubano de Ciencias Pedagógicas.

Caro, B. (2014). Utilización de TIC, Competencias Básicas y Calidad de la Educación. Revista Virtual Universidad Católica del Norte. 42 (2). 4-37. Recuperado de www.redalyc.org/service/redalyc/downloadPdf/1942/194230899002/6

Chawla, D. \& Sodhi, N. (2011). Metodología de la investigación: Conceptos y casos. Nueva Delhi: Vikas Publishing House.

Gutiérrez, C. (2018). ¿Están preparados nuestros universitarios para trabajar en las empresas del futuro? Revista AECA No 113. 47 - 50. Recuperado de http:// www.aeca1.org/revistaeca/revista113/113.pdf

Hernández, E.J, Barbera, N, Vega, A.A \& Bru, O.E (2018). Aprendizaje basado en el problema: una estrategia pedagógica para la formulación de proyectos de investigación en el aula. En Y. del Valle (Ed.), Tendencias en Investigación Universitaria. Una visión desde Latinoamérica. Vol. III. (pp. 146-165). Falcón, Venezuela: Fondo Editorial Universitario Servando Garcés Recuperado de https://investigacionuptag.wordpress.com/

Huh, J., Delorme, D. E., \& Reid, L. N. (2006). Perceived third-person effects and consumer attitudes on preventing and banning DTC advertising. Journal of Consumer Affairs, 40, 90-116.

Infante, Ch. (2014). Propuesta pedagógica para el uso de laboratorios virtuales como actividad complementaria en las asignaturas teóricoprácticas. RMIE. 19 (62), 917-937. Recuperado de http://www.comie.org.mx/documentos/rmie/v19/ n062/pdf/62012.pdf

López, R. \& Hernández, M. (2016). Principios para elaborar un modelo pedagógico universitario basado en las TIC. Estado del arte. Revista Digital de Ciencia, Tecnología e Innovación. 3 (4), 575 - 593. Recuperado de http://45.238.216.13/ ojs/index.php/EPISTEME/article/view/450/241

López, L (2001). El desarrollo de las habilidades de investigación en la formación inicial del profesorado de Química. (Tesis doctoral). Universidad de Cienfuegos, Cienfuegos, Cuba.

Martínez, D. \& Márquez, D. L. (2014). Las habilidades investigativas como eje transversal de la formación para la investigación. Revista Tendencias Pedagógicas. Vol. 24. 347-360. Recuperado de https://revistas.uam.es/tendenciaspedagogicas/article/ view/2110 
Montes de Oca, N. \& Machado, E. F. (2009). El desarrollo de habilidades investigativas en la educación superior: un acercamiento para su desarrollo. Humanidades Médicas, 9(1). 1-28 Recuperado de http://scielo.sld.cu/scielo.php?script=sci_ arttext\&pid=S1727-81202009000100003\&lng=es\&tlng=es

Orozco, G. H, Sosa, M. R. \& Martínez, F. (2018). Modelos didácticos en la Educación Superior: Una realidad que se puede cambiar. Revista Profesorado. 22 (2). 447 - 469. Recuperado de https://recyt.fecyt.es/index.php/profesorado/article/ view/66382/41131

Tiburcio, M.L, Godínez, R. \& Aguilar, M. J. (2018). Mejora organizacional basada en la gestión del conocimiento para PYMES de base tecnológica: Estudio de caso de la empresa XYZ. En Y. del Valle (Ed.), Tendencias en Investigación Universitaria. Una visión desde Latinoamérica. Vol. III. (pp. 28-43). Falcón, Venezuela: Fondo Editorial Universitario Servando Garcés. Recuperado de https://investigacionuptag.wordpress.com/ 